\title{
September 2013 Critical Care Case of the Month: Revenge of the Pharaohs
}

\author{
Robert A. Raschke, MD \\ Elijah Poulos, MD
}

\author{
Banner Good Samaritan Regional Medical Center \\ Phoenix, AZ
}

\section{History of Present IIIness}

The patient was a 68 year-old man, admitted to our ICU through the emergency room (ER) in July 2013 with suspected urinary tract origin sepsis.

The patient was evaluated in ER by the ICU team. He was in his usual state of general good health until he visited his primary care physician for what he felt was a left inguinal hernia, and underwent a prostate examination, four days previously. The patient associated this prostate examination with the onset of fevers and chills that began the next morning. He was seen in an urgent care center where he was told his urinalysis was normal, and antibiotics were not prescribed. Over the intervening 3 days, he suffered recurrent fevers, had vomited three times, and had one diarrheal bowel movement. Earlier on the day of presentation, he had been mowing his lawn (in $>100^{\circ} \mathrm{F}$ environment) and had become a little dizzy. His wife, a retired nurse, finally convinced him to report to the ER.

He denied dysuria, urinary frequency or urgency, headache, sore throat, cough, or abdominal pain.

\section{$\mathrm{PMH}, \mathrm{SH}, \mathrm{FH}$}

He had a prior history of hypertension, gastroesophageal reflux, gout and hypercholesterolemia. He drank alcohol about twice a month and did not smoke.

There was no family history of illnesses.

\section{Medications}

- Atorvastatin

- Allopurinol

- Hydrochlorothiazide

- Lisinopril

- Temazepam

\section{Physical Exam}


On ER triage, his temperature was $41.2^{\circ} \mathrm{C}$, but vitals at the time of our initial examination were temp $38.2^{\circ} \mathrm{C}, \mathrm{HR} 93$ beats/min, BP 103/48 mm Hg, and respiratory rate 20 breaths/min. He was awake and alert, but made a few errors while relating his history - for instance, he initially answered yes when asked if he had a headache, then corrected himself and said no - he meant he had a fever. He was actively rigoring. HEENT exam was unrevealing. He had no lymphadenopathy. His lungs were clear. His abdomen was soft and nontender. $\mathrm{He}$ had a sliding left inguinal hernia that was not tender. None of his joints were acutely inflamed. His prostate was not enlarged or tender to palpation. He had no focal neurological deficits.

\section{Laboratory}

Pertinent laboratory values in the ER:

- WBC: $7.7 \times 10^{9} / \mathrm{L}$

- Hematocrit: $38.4 \%$

- Sodium: $131 \mathrm{me} / \mathrm{L}$

- Potassium: $3.1 \mathrm{me} / \mathrm{L}$

- BUN:28 g/dL

- Creatinine: $1.3 \mathrm{mg} / \mathrm{dL}$

- Lactate: $2.1 \mathrm{mMol} / \mathrm{L}$.

The rest of his admission labs and urinalysis were unremarkable.

\section{Chest Radiography}

His initial portable chest x-ray is shown in Figure 1.

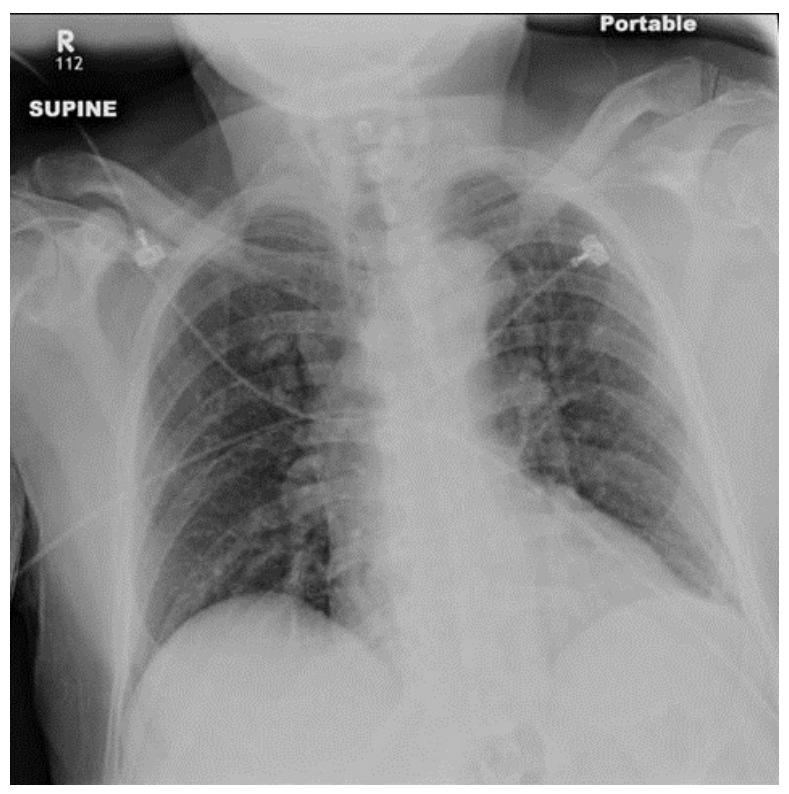

Figure 1. Initial portable chest x-ray. 
Which of the following is the likely cause of his fever?

1. Prostatitis exacerbated by digital rectal exam

2. Right middle lobe pneumonia

3. Urinary tract infection

4. All of the above

5. None of the above 


\section{Correct! \\ 5. None of the above}

Urinary tract infections and pneumonia are two very frequent causes of fever. Prostatitis can occur acutely or chronically and may be a source of fever. However, the patient really has no symptoms pointing to any of these as a source of fever. Furthermore, his physical exam, chest x-ray shows, and urinanalysis show no focal abnormalities suggesting any of these as a source of infection.

The ER staff had placed a Foley catheter and administered ceftriaxone and ciprofloxacin for a presumed urinary tract infection. They had also administered three liters of intravenous $0.9 \%$ saline, but the patient's blood pressure fell to $87 / 41 \mathrm{mmHg}$.

We transferred the patient to the ICU, continued intravenous hydration and electrolyte replacement, and changed the antibiotics to pipericillin/tazobactam plus vancomycin, and discontinued his Foley catheter.

What is the best next step to obtain a diagnosis in this patient?

1. Blood cultures

2. Bronchoscopy with bronchoalveolar lavage

3. Chest, abdominal and pelvic CT scan

4. Obtain additional history and a more thorough physical examination

5. Urine culture 


\section{Correct!}

\section{Obtain additional history and a more thorough physical examination}

Cultures may eventually provide a diagnosis and were obtained. However, they will not give a diagnosis at this time. CT scans of the chest, abdomen and pelvis were also obtained by the ER physicians and were interpreted as normal. The lack of symptoms or signs pointing to pathology in these areas makes CT scanning a low yield test. Bronchoscopy with bronchoalveolar lavage is not indicated and very unlikely to provide a diagnosis in a patient who has no thoracic symptoms, a normal chest $\mathrm{x}$-ray and a normal CT scan.

After consideration, we decided that we didn't have a tenable diagnosis and must be missing something. We took more history. The only recent travel history outside Arizona was a sailing trip in San Diego two months previously. The patient reported no history of camping, hunting, tick bites (although his wife interjected that "mosquitoes love him"), no exposure to animals except his pet cat, no cat bites or scratches, no ingestion of unpasteurized diary products (such as Mexican cheese) and no recent exposure to anyone else who was ill. Recalling his possible confusion when previously asked about headaches, he was asked again. He reported that he had been having severe headaches and photophobia for the past four days. His wife stated that she had wondered why he had originally denied headaches since he had been asking her for ice-packs for his head for the past three days. A more thorough physical examination was repeated and there no additional findings discovered including nuchal rigidity (stiff neck) and no focal neurologic signs.

Which of the following is (are) the best next step(s) in management of the patient?

1. CT scan of the head

2. Needle biopsy of the prostrate

3. Lumbar puncture (LP)

4. 1 and 3

5. All of the above 


\section{Correct! \\ 3. Lumbar puncture (LP)}

There are no signs or symptoms of prostatitis and a needle biopsy of the prostrate is inappropriate. It was common to perform a CT scan before a LP to exclude a space occupying lesion and potential brainstem herniation. However, in adults with suspected meningitis, clinical features can be used to identify those who are unlikely to have abnormal findings on CT of the head (1). These include an age of at least 60 years, immunocompromise, a history of central nervous system disease, and a history of seizure within one week before presentation, as well as the following neurologic abnormalities: an abnormal level of consciousness, an inability to answer two consecutive questions correctly or to follow two consecutive commands, gaze palsy, abnormal visual fields, facial palsy, arm drift, leg drift, and abnormal language (e.g., aphasia).

Based on his slight confusion an LP was performed which was initially colorless, but became pink-tinged as cerebrospinal fluid was withdrawn - it did not clear as further samples were acquired. There were 13,093 RBC, 294 WBC (17\% segmented, $70 \%$ lymphocytes, and $13 \%$ monocytes), glucose was 61 and protein 123. Gram stain is negative.

Which of the following antibiotics should be empirically administered?
1. Acyclovir
2. Ampicillin
3. Ceftriaxone
4. Vancomycin
5. All of the above 


\section{Correct! \\ 5. All of the above}

Given the clinical situation and the abnormal CSF findings, meningitis is a possibility. Empiric antibiotic treatment is indicated pending the results of cultures. He was administered ceftriazone $2 \mathrm{gm}$ every 12 hours, vancomycin $15 \mathrm{mg} / \mathrm{kg}$ every 12 hours (empirical treatment for bacterial meningitis), ampicillin 4 million units every 6 hrs (to cover Listeria meningitis since he was $>60$ years of age) and acyclovir 10mg/kg every 6 hours (to cover Herpes simplex encephalitis).

Over the next 48 hours, the patient had recurrent fevers and chills and intermittently became agitated and confused. Progressive lethargy intervened, and he was intubated for airway protection. Bacterial blood cultures and CSF culture were negative. CSF Herpes simplex virus polymerase chain reaction (PCR), and coccidioidomycosis complement fixation were negative, as were serological tests for enterovirus, Western and Eastern equine, St. Louis and California encephalitis viruses.

Which of the following is the most likely cause of the patient's clinical picture?

1. Cat scratch fever

2. Guillain-Barré syndrome

3. Japanese encephalitis virus

4. Murray Valley encephalitis virus

5. West Nile encephalitis 


\section{Correct! \\ 5. West Nile encephalitis}

The only manner to differentiate between causes of encephalitis/meningitis is diagnostic and serological laboratory tests to identify the specific pathogen causing the symptoms. The CSF IgM serology for West Nile Virus (WNV) was positive at 5.97 (normal < 0.9). Japanese encephalitis virus and Murray Valley encephalitis would present similarly but are limited to Asia (2). Guillain-Barré could initially present similarly but there is no history of ascending muscle weakness. Furthermore, there is an absence of the characteristic markedly elevated CSF protein level (100-1000 mg/dL), without an accompanying increased cell count (absence of pleocytosis) seen in Guillain-Barré. Cat scratch fever can very rarely present with encephalitis but is unlikely. The patient's antibiotics were discontinued; he was extubated after on his fourth hospital day, and discharged on his sixth.

WNV is a mosquito-borne zooinotic arbovirus (3). It was first described in the Western hemisphere in 1999 in New York City (NYC). Simultaneous clusters of unexplained meningoencephalitis struck NYC hospitals, and fatal bird infections struck the Queens zoo - both found to be caused by WNV. Since then, WNV has spread westward to the Pacific coast, and into Canada and Latin America. The year 2012 was the worst year on record in the US with 268 deaths - the state of Texas was the most hard-hit.

WNV should be suspected in any patient presenting with an acute febrile disease or meningitis, who has been exposed to mosquitoes or blood transfusion in a state with active WNV epidemiology (3). It presents primarily in the summer as mosquitoes become active. Eighty percent of cases are asymptomatic, and $20 \%$ present with fever, headache, myalgias, arthralgias, gastrointestinal upset, and rash. Less than one percent suffers meningitis or meningoencephalitis as in our patient. Acute flaccid paralysis can occur secondary to a poliomyelitis-like infection of the anterior horn cells of the spinal column, or from Guillain-Barré syndrome. WNV can be diagnosed by serum or CSF IgM antibodies that are usually detectable 3-8 days after initial symptoms. Most patients recover, but $10 \%$ of patients with neuroinvasive disease die, and neurological sequelae can persist. In those that recover, fatigue can persist for months.

The 2013 WNV season is still early, but as of July the CDC is reporting 31 cases from 14 states - mostly in the Midwest and the Southwest (3). Thirty-five percent of the reported cases were neuroinvasive, with 3 fatalities thus far.

One of my mentor's favorite sayings is that "history-taking is never completed" this case again confirms his wisdom.

\section{References}


1. Hasbun R, Abrahams J, Jekel J, Quagliarello VJ. Computed tomography of the head before lumbar puncture in adults with suspected meningitis. N Engl J Med. 2001;345(24):1727-33. [CrossRef] [PubMed]

2. Rossi SL, Ross TM, Evans JD. West Nile virus. Clin Lab Med. 2010; 30(1): 47-65. [CrossRef] [PubMed]

3. http://www.cdc.gov/westnile/index.html (accessed 8/9/13). 